\title{
Foveal electroretinograms and choroidal perfusion characteristics in fellow eyes of patients with unilateral neovascular age-related macular degeneration
}

\author{
Juancho F C Remulla, Alexander R Gaudio, Sumiko Miller, Michael A Sandberg
}

\begin{abstract}
Aims/Background-A prolonged choroidal filling phase on fluorescein angiography has been reported to be a common finding and associated with visual function abnormalities in patients with age-related macular degeneration (AMD). This investigation was carried out to determine whether this perfusion defect was related to the slowing of foveal cone electroretinogram (ERG) implicit time seen in patients with AMD.
\end{abstract}

Methods-Fluorescein angiograms and foveal cone ERGs were evaluated in the fellow eyes of 67 patients with unilateral neovascular AMD.

Results-Twenty eight (42\%) of the eyes had a choroidal perfusion defect. ERG implicit times averaged $1 \mathrm{~ms}$ slower $(p=0.0167)$ and were more likely to be delayed $(p=0.0078)$ in eyes with abnormal choroidal perfusion than in eyes with normal choroidal filling; significant relations were found also after controlling for age. ERG implicit time was also inversely related to ETDRS visual acuity and positively related to the extent of macular drusen; and the latter showed a borderline significant tendency to be more prevalent in eyes with prolonged choroidal perfusion. However, an association of a delayed ERG implicit time with prolonged choroidal filling remained after controlling for age, acuity, and the extent of drusen.

Conclusion-These findings further establish prolonged choroidal perfusion as a common finding in AMD and link it to retinal malfunction.

(Br f Ophthalmol 1995; 79: 558-561)

Berman-Gund

Study of Retinal

Degenerations,

Harvard Medical

School, Massachusetts

Eye and Ear

Infirmary, Boston,

USA

J F C Remulla

A R Gaudio

$S$ Miller

M A Sandberg

Correspondence to:

Dr Michael A Sandberg,

Berman-Gund Laboratory,

Massachusetts Eye and Ea

Infirmary, 243 Charles

Street, Boston, MA 02114

USA.

Accepted for publication

10 January 1995 of cone and rod dark adation 6 It has thought to reflect either an attenuated choriocapillaris, seen histologically, ${ }^{7-9}$ together with a diffusely thickened Bruch's membrane, ${ }^{13}$ or a thickened Bruch's membrane alone. ${ }^{2}$
A prolonged choroidal filling phase on flut 100 cases $^{1}$ and $33 \%$ of 126 cases $^{2}$ with age(AMD), and AMD. ${ }^{3}$ This perfusion abnormality is associated with patchy elevations of dark adapted threshold, ${ }^{4}$ an increased risk for developing
We recently reported that fellow eyes of patients with unilateral neovascular AMD have on average foveal cone ERG amplitudes that are normal, but implicit (peak) times that are slower than normal. ${ }^{10}$ Since a defect in choroidal filling is apparently common in patients with $\mathrm{AMD}^{1-3}$ and is associated with visual dysfunction, ${ }^{46}$ we investigated whether this angiographic finding was also related to the slowed foveal ERG seen in the fellow eyes of patients with the unilateral neovascular form.

\section{Methods}

We evaluated the fellow eyes of 67 patients (ages 61 to 89) with unilateral neovascular AMD. These patients were part of a larger group with unilateral neovascular AMD who are being followed prospectively with foveal cone ERGs and other tests of cone function to predict who will develop a choroidal neovascular membrane in the fellow eye; demographic characteristics and ocular findings of this larger group have been described previously. ${ }^{11}$ Eligibility criteria for the present report included a corrected Snellen visual acuity of $20 / 60$ or better, sufficiently clear media to allow detailed evaluation of the fundus, macular drusen, and no sign of other retinal disease in the study eye.

These study eyes had a foveal cone ERG recorded and had angiograms of clear, readable quality throughout the transit. The angiograms were read by two of the authors (JCR and ARG), masked with respect to results of foveal cone electroretinography, to identify those angiograms with a prolonged choroidal filling phase; the latter was characterised by a non-uniform fluorescence extending over at least 5 disc diameters of the posterior pole persisting through the onset of the venous phase of the retinal circulation. ${ }^{1}$

Foveal cone ERGs were elicited with a $4^{\circ}$ white stimulus flickering at $42 \mathrm{~Hz}$ presented by a hand held, dual beam stimulator ophthalmoscope (Maculoscope, Doran Instruments, Littleton, MA, USA) as previously described. ${ }^{10} 12$ Responses were monitored with a contact lens electrode, amplified, filtered, digitised, summed, and quantified by Fourier analysis with respect to amplitude and phase; phase was then converted to implicit time (that is, time interval from stimulus onset to the corresponding cornea positive response peak). 
Table 1 Patient age and study eye characteristics by category of choroidal filling

\begin{tabular}{llll}
\hline Variable & Normal filling & Prolonged filling & $p$ Value \\
\hline Age & $72 \cdot 3(1 \cdot 1)$ years & $74 \cdot 3(1 \cdot 4)$ years & $0 \cdot 2816 \dagger$ \\
ETDRS acuity & $38 \cdot 8(1 \cdot 3)$ letters & $37 \cdot 2(1 \cdot 6)$ letters & $0 \cdot 4428 \dagger$ \\
Lens opacity & $58 \%$ of cases & $56 \%$ of cases & $0 \cdot 8818 \ddagger$ \\
Extent of drusen & $3 \cdot 1(0 \cdot 6) \%^{\star}$ & $3.9(0 \cdot 9) \%^{\star}$ & $0 \cdot 0509$ 物
\end{tabular}

^Mean (SE); the ETDRS acuities transposed to the Snellen scale were 20/27 for patients with normal choroidal filling and $20 / 29$ for patients with prolonged choroidal filling.

$\dagger$ Student's $t$ test. $¥ \chi^{2}$ test. $\lceil t$ test for unequal variances based on log percent.

Table 2 Multiple regression of foveal cone ERG implicit time on age and category of choroidal filling

\begin{tabular}{lllll}
\hline Term & Estimate & $S E$ & t Ratio & p Value \\
\hline Age & 0.11 & 0.03 & 3.32 & 0.0015 \\
Choroidal filling & 1.02 & 0.47 & 2.17 & 0.0336 \\
\hline
\end{tabular}

$\star$ Normal $=0$, prolonged $=1$.

Table 3 Distribution of cases by foveal ERG implicit time and category of choroidal filling

\begin{tabular}{llll}
\hline & Normal filling & Prolonged filling & Total \\
\hline Normal implicit time & 28 & 11 & 39 \\
Delayed implicit time & 11 & 17 & 28 \\
Total & 39 & 28 & 67 \\
\hline
\end{tabular}

$\star 31$ to $38 \mathrm{~ms}$

Possible confounding factors for the relation of foveal cone ERG implicit time to choroidal perfusion characteristics were also evaluated in the study eyes: we measured their visual acuity on an ETDRS chart, ${ }^{11}$ coded the presence of a lens opacity in non-pseudophakic eyes, and estimated their area of macular drusen from fundus photographs ${ }^{13}$ without reference to the ERG and angiographic results. Since the frequency distributions of drusen area and foveal

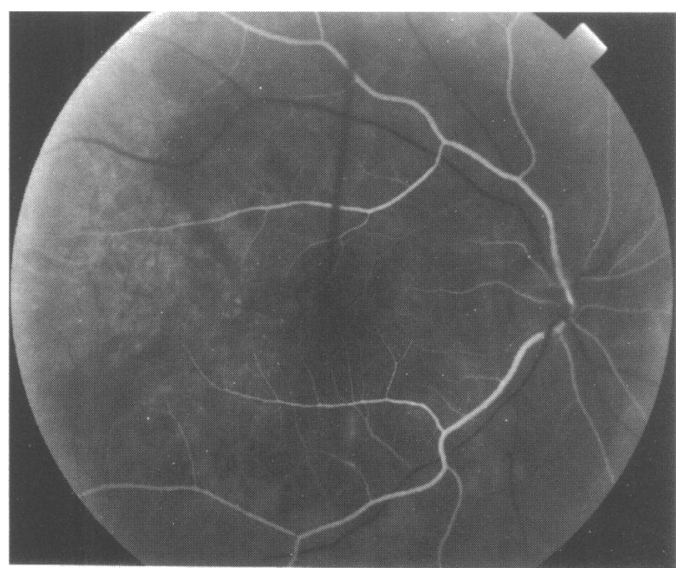

Fig $1 A$

Figure 1 Fluorescein angiogram from the fellow eye of a 66-year-old woman with unilateral neovascular age-related macular degeneration. The fluorescein angiogram shows persistence of a patchy choroidal

fluorescence in the posterior pole at the onset of the

venous phase. This eye had a foveal cone ERG implicit time of $39.4 \mathrm{~ms}$.

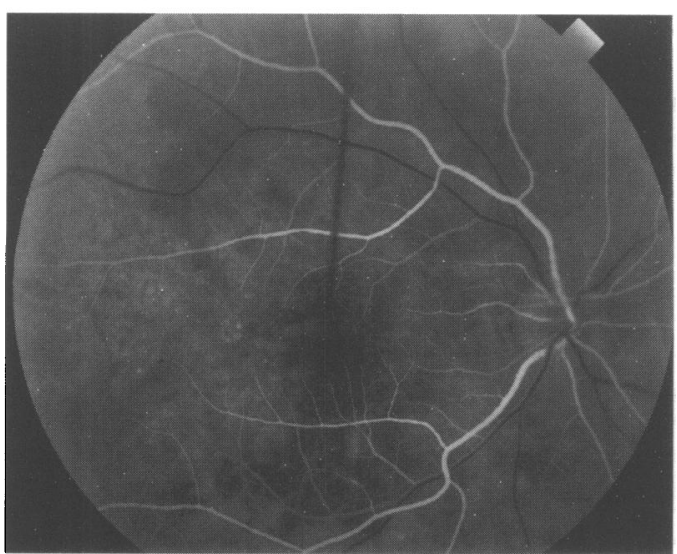

Fig $1 B$ cone ERG amplitude were positively skewed, both measures were converted to common logarithms to approximate better a normal distribution. This research followed the tenets of the Declaration of Helsinki and was approved by the internal review boards of the Massachusetts Eye and Ear Infirmary and Harvard Medical School.

\section{Results}

Angiographic evidence of prolonged choroidal filling was demonstrated in 28 of the 67 eyes $(42 \%)$. Patients with prolonged choroidal filling were similar to those with normal choroidal filling with respect to mean age, mean ETDRS acuity in the study eye, and the prevalence of a lens opacity in the study eye (Table 1). On the other hand, patients with prolonged choroidal perfusion had a tendency to have more extensive macular drusen (Table 1).

The mean (SE) for foveal cone ERG implicit time was $38.3(0.4) \mathrm{ms}$ for patients with a prolonged choroidal filling phase and $37 \cdot 0(0 \cdot 3) \mathrm{ms}$ for those with normal choroidal filling; these means were significantly different ( $t$ test, $\mathrm{p}=0.0167$ ). If we controlled for the positive relation between ERG implicit time and age by multiple regression, ${ }^{10}$ then mean implicit times for the two groups of patients remained significantly different (Table 2 ). If we divided the patients into those with a delayed ERG implicit time (that is, greater than $38 \mathrm{~ms}^{14}$ ) and those with a normal implicit time, then the presence of a delayed implicit time was significantly related to the presence of prolonged choroidal filling by contingency table analysis (Table 3; $\mathrm{p}=0.0078$ ) and by logistic regression controlling for age $(p=0.0161)$. If a patient had a prolonged perfusion time, then the average odds were 3.9 to 1 that the patient also had a delayed implicit time. An example of a fluorescein angiogram with abnormal choroidal filling from a patient with a delayed ERG implicit time is illustrated in Figure 1.

We also found that a slower or delayed implicit time was inversely related to ETDRS acuity (0.0098 and 0.0229, respectively) and positively related to the extent of drusen $(p=0.0026$ and $p=0.0375$, respectively) when controlling for age; there was no relation of a slower or delayed implicit time to the presence

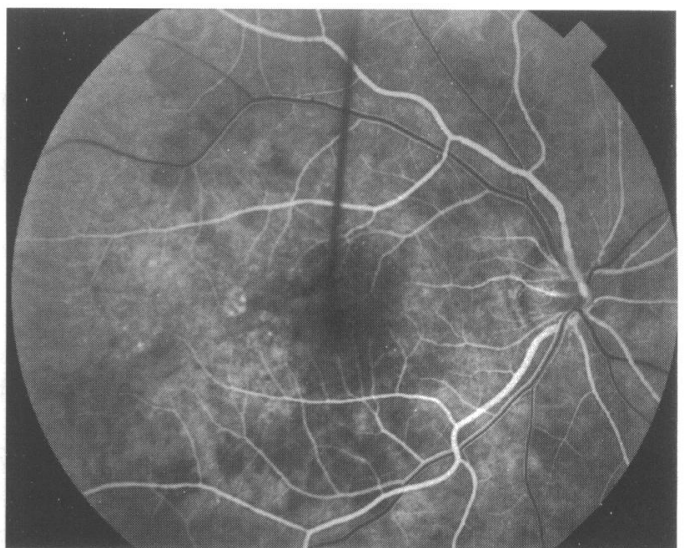

Fig $1 C$ 


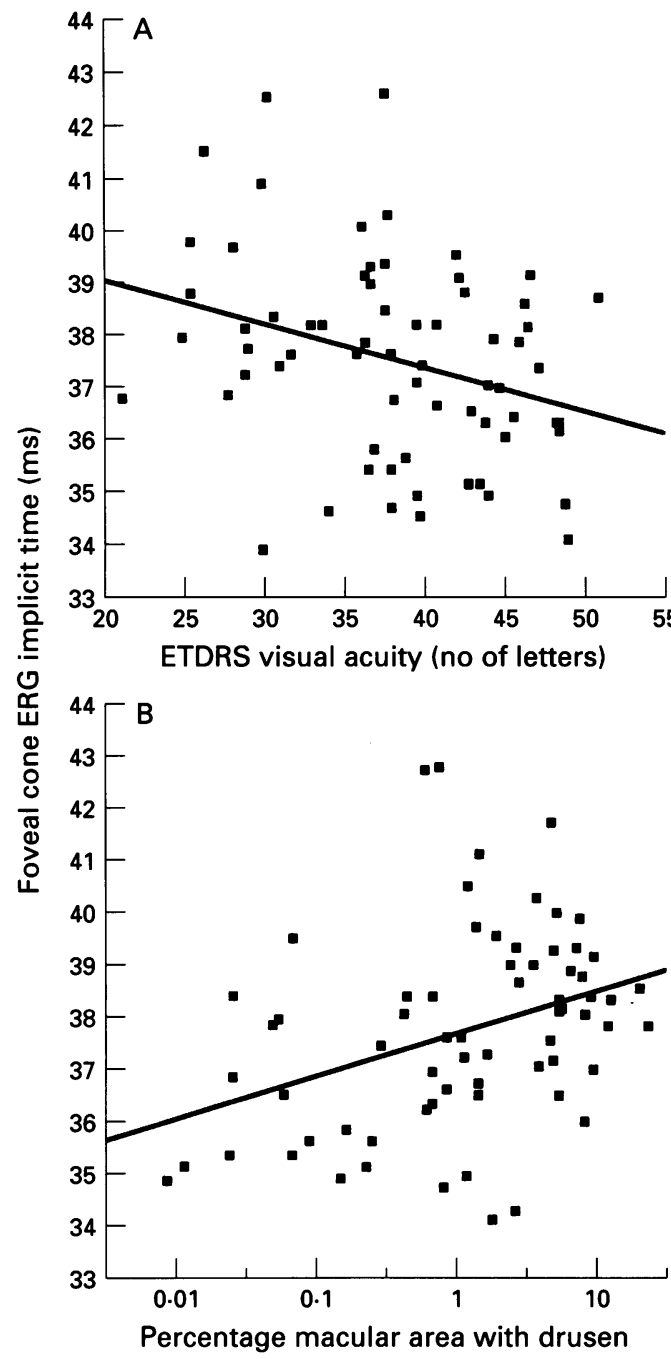

Figure 2 Age adjusted regression of foveal cone ERG implicit time on ETDRS acuity $(A)$ and on percentage area of the macula (central $15^{\circ}$ ) with drusen on a logarithmic scale (B) based on the fellow eyes of 67 patients with unilateral neovascular AMD. An ETDRS acuity of 45 letters $=20 / 20{ }^{11}$ The area of drusen was calculated by multiplying the number of drusen by the mean drusen area. 13

of a lens opacity $(0.9725$ and 0.6530 , respectively) when controlling for age. The age adjusted regression of implicit time on acuity and on the percentage area of drusen are shown in Figure 2. Despite these associations, implicit time was more likely to be delayed in patients with prolonged choroidal filling even when controlling for age, acuity, and area of drusen $(p=0.0299)$. There was no difference between the groups with respect to foveal cone ERG amplitudes $(p=0.5804)$ : the values were $-0.62(0.03) \log \mu \mathrm{V}$ (geometric mean, 0.24 $\mu \mathrm{V})$ for the patients with prolonged choroidal filling and $-0.60(0.02) \log \mu \mathrm{V}$ (geometric mean, $0.25 \mu \mathrm{V}$ ) for the patients with normal choroidal filling.

\section{Discussion}

We found that the percentage of fellow eyes of patients with unilateral neovascular AMD with a prolonged choroidal filling phase on fluorescein angiography $(42 \%)$ fell within the range of previously reported values for eyes with AMD. ${ }^{1-3}$ By combining our results with those previously published, the prevalence of this defect becomes 166 of 399 cases (42\%), further establishing it as a common angiographic sign in AMD.

Prolonged choroidal perfusion was associated with a foveal cone ERG implicit time that was slower and more likely to be delayed. This adds an objective measure of retinal malfunction to the previously described psychophysical abnormalities of impaired sensitivity in the dark and slowed recovery during dark adaptation for AMD eyes with this perfusion defect. $^{46}$ This relation implicates an abnormality of the choriocapillaris and/or Bruch's membrane, directly or indirectly, in slowing the foveal cone ERG in this disease.

We also noted that ERG implicit time was inversely related to ETDRS visual acuity and positively related to the extent of macular drusen in our patients. The second finding may help to account for the previous observation that foveal cone ERG implicit time was slower in fellow eyes with normal visual acuity of patients with unilateral neovascular $\mathrm{AMD}^{10}$ many of whom have extensive drusen, than in normal subjects, who have minimal (if any) drusen. Our patients with prolonged choroidal perfusion also showed a borderline tendency to have more extensive drusen. Nevertheless, prolonged choroidal perfusion remained a significant predictor of a delayed ERG implicit time even after controlling for acuity and extent of drusen. The association of ERG implicit time to prolonged choroidal perfusion also could not be explained by a difference in media clarity between the patients with and without the perfusion defect, because the two groups had comparable prevalences of lens opacity and because implicit time was unrelated to the presence of a lens opacity.

Foveal cone ERG amplitude was unrelated to the choroidal perfusion characteristics of the study eyes. A delayed cone ERG response with normal amplitude may be seen in the full field cone ERG of some patients with central retinal vein occlusion (Sandberg and Gaudio, unpublished observations) and in the foveal cone ERG of some patients with diabetic macular oedema. ${ }^{15}$ Conceivably, a vascular component may be similarly implicated in eyes with prolonged choroidal filling. It is possible that the angiographic finding of prolonged choroidal perfusion in patients with $A M D$ is reflective of outer retinal ischaemia, resulting in foveal cone ERGs of normal amplitude but delayed implicit time.

This work was supported by grants from the National Eye Institute (EY08398), the Foundation Fighting Blindness, Baltimore, MD, the Massachusetts Lions Eye Research Fund Inc, and the Lawrence $J$ and Anne Cable Rubenstein Foundation, Boston, MA, USA.

1 Pauleikhoff D, Chen JC, Chisholm IH, Bird AC. Choroidal perfusion abnormality with age-related Bruch's membrane change. Am f Ophthalmol 1990; 109: 211-7.

2 Staurenghi G, Bottoni F, Lonati C, Autelitano A, Orzalesi N. Drusen and 'choroidal filling defects': a cross-sectional survey. Ophthalmologica 1992; 205: 178-86.

3 Boker T, Fang T, Steinmetz R. Refractive error and choroidal perfusion characteristics in patients with choroidal peovascularization and age-related macular degeneration. German $₹$ Ophthalmol 1993; 2: 10-3.

4 Chen JC, Fitzke FW, Pauleikhoff D, Bird AC. Functional loss in age-related Bruch's membrane change with loss in age-related Bruch's membrane change with 33: $334-40$. 
5 Piguet B, Palmvang IB, Chisholm IH, Minassian D, Bird AC. Evolution of age-related macular degeneration with choroidal perfusion abnormality. Am F Ophthalmol 1992; 113: $657-63$

6 Steinmetz AU, Haimovici R, Jubb C, Fitzke FW, Bird AC. Symptomatic abnormalities of dark adaptation in patients
with age-related Bruch's membrane change. $\mathrm{Br} f$ with age-related Bruch's men

7 Tso MOM. Pathogenetic factors of aging macular degeneration. Ophthalmology 1985; 92: 628-35.

8 Sarks SH, Sarks J, Killingsworth C. Evolution of geographic atrophy of the retinal pigment epithelium. Eye 1988; 2: 552-77.

9 Olver J, Pauleikhoff D, Bird AC. Morphometric analyses of age-changes in the choriocapillaris. Invest Ophthalmol Vis Sci 1990; 31 (suppl): 47.

10 Sandberg MA, Miller S, Gaudio AR. Foveal cone ERGs in fellow eyes of patients with unilateral neovascular age- related macular degeneration. Invest Ophthalmol Vis $\mathrm{Sc}$ 1993; 34: 3477-80.

11 Sandberg MA, Gaudio AR, Miller S, Weiner A. Iris pigmentation and extent of disease in patients with neovascular age-related macular degeneration. Invest Ophthalmol Vis Sci 1994; 35: 2734-40.

12 Weiner A, Sandberg MA. Normal change in the foveal cone ERG with increasing duration of light exposure. Invest ERG with increasing duration of light
Ophthalmol Vis Sci 1991; 32: 2842-5.

13 Tolentino MJ, Miller S, Gaudio AR, Sandberg MA. Visual field deficits in early age-related macular degeneration. Vision Res 1994; 34: 409-13.

14 Sandberg MA, Jacobson SG, Berson EL. Foveal cone electroretinograms in retinitis pigmentosa and juvenile macular degeneration. Am f Ophthalmol 1979; 88: 702-7.

15 Brodie SE, Sperber DE, Hope-Ross M. Focal ERG phaselag diabetic macular edema. Invest Ophthalmol Vis $\mathrm{Sci}$ 1993; 34 (suppl): 1179.

\section{History of ophthalmology}

\section{Discovering the uses of the ophthalmoscope}

In the early years after Helmholtz invented the ophthalmoscope, the even greater task of discovering what was normal and what was pathological began. Not that the instrument was rapidly accepted - to quote the editor of the Medical Times, it was 'ridiculed and denounced' by many physicians as a dangerous toy! Jabez Hogg of the Westminster Ophthalmic Hospital worked hard to redeem this situation by publishing a book on its use. However, those attending the Ophthalmological Congress in Brussels in 1857 debated its utility at length.

Summaries of the normal and abnormal appearances of the retina began to appear in the literature. Opacities in the lens were noted, and Williams describes the 'beautiful opaque streaks' with enthusiasm. Floating bodies in the vitreous were seen 'bounding and darting about' and were considered common, although their cause was a mystery.

By 1865 , funduscopy was confidently used in the diagnosis of renal disease. For example, 'Mary Ann' was a sewing machinist who had undergone much hardship. Her sight had begun to fail since Christmas 1864 when she had 'sat all day in wet clothes', and she presented to $\mathrm{Mr}$ Hulke. On ophthalmoscopy, her retinal veins were 'turgid', and grey-white patches were visible. Kidney disease was diagnosed, and confirmed by the finding of albumin and casts in the urine. Mr Hulke sent 'Mary Ann' away with some muriate of iron, and exhorted his colleagues to remember the use of funduscopy in renal disease. Dr Hughlings Jackson agreed with this point, while stressing the association of fundal changes with cerebrovascular accidents. A pithy description of a patient with retinal haemorrhages who was 'in a ripe condition for extensive cerebral apoplexy', but 'also ready to die in many other ways' followed. It is hoped that this opinion was not communicated to the patient, or at least that his alleged slight deafness saved him from hearing it!

Later, in the early 1900 s, more detailed investigation of 'flame-shaped haemorrhages' and arteriovenous nipping took place, and the connection with hypertension firmly established. Understandably, some misdirections occurred:
Bardsley differentiated the signs of arteriosclerosis from hypertension, and believed that the retinal signs could appear and disappear rapidly. Armed with a large supply of adrenaline chloride and his house officer he set out to prove this on his patients. After injecting enough adrenaline to secure a rise in blood pressure of at least $45 \mathrm{~mm} \mathrm{Hg}$ - an effect which could be achieved in an ethics committee chairman merely by reading this account - the retina was examined. Bardsley reported that fullness of veins and AV nipping was seen - just as he had previously noted in cases of toxaemia, and that it disappeared when the blood pressure fell. Indeed, having tried this on all the patients, he felt that it should be possible to indicate the blood pressure merely from funduscopy.

Others felt that even established arteriosclerosis was reversible. Crofton described a 60-year-old man with small, tortuous retinal arteries and severe AV nipping. Advised of his grave prognosis, the patient went on a sea voyage. On his return 2 months later, Crofton reported that all silver wiring was gone, and the AV nipping had considerably lessened.

Not all physicians believed in the prognostic implications of high blood pressure, although many prohibited the use of the eyes, feeling that the strain of vision might worsen the disease. Adams described a 64-year-old with an arterial pressure of 250 and a host of flame-shaped haemorrhages, who lived a further 4 years. 'Why treat?' insisted Adams, as hypertension is a sign to which the patient does not object.

Doubtless these physicians would be fascinated to hear about the developments that have occurred, although if Crofton did cure the retinal signs of hypertension, then it seems that some valuable knowledge has been lost.

F ROMAN

Adams $\mathrm{PH}$. Influence of vascular disease of the retina on prognosis of life. $B r$ F Ophthalmol 1917; 1: 55

Anonymous. Medical and surgical practice. Medical Times and Gazette 1865; Nov 18: 551 .

Bardsley PC. The retinal signs of arteriosclerosis compared with those due simply to high blood pressure. Br $\mathcal{f}$ Ophthalmol 1917 ; 1: 239

Crofton WM. Can advanced arteriosclerosis be reversible? $B M \mathcal{F}$

1910; Aug 6.
Williams E. The ophthalmoscope. Medical Times and Gazette 1854; 9: 7-11. 